\title{
Low-dose $(0.01 \%)$ atropine eye-drops to reduce progression of myopia in children: a multicentre placebo-controlled randomised trial in the UK (CHAMP-UK) - study protocol
}

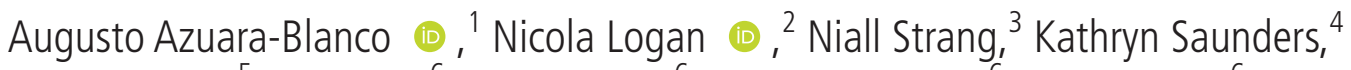 \\ Peter M Allen, ${ }^{5}$ Ruth Weir, ${ }^{6}$ Paul Doherty, ${ }^{6}$ Catherine Adams, ${ }^{6}$ Evie Gardner, ${ }^{6}$ \\ Ruth Hogg (1) , ${ }^{1}$ Margaret McFarland, ${ }^{7}$ Jennifer Preston, ${ }^{8}$ Rejina Verghis, ${ }^{6}$ \\ James J Loughman (1), ${ }^{9}$ Ian Flitcroft, ${ }^{10}$ David A Mackey, ${ }^{11}$

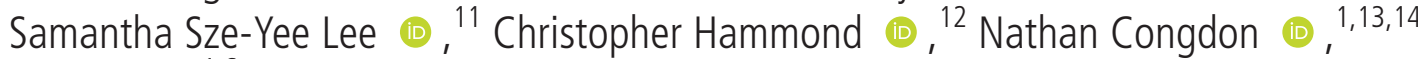 \\ Mike Clarke ${ }^{1,6}$
}

For numbered affiliations see end of article.

\section{Correspondence to} Professor Augusto AzuaraBlanco, Centre for Public Health, Queen's University Belfast, Belfast BT12 6BA, UK a.azuara-blanco@qub.ac.uk

Received 26 June 2019 Revised 9 September 2019 Accepted 5 October 2019 Published Online First 25 October 2019
Check for updates

(C) Author(s) (or their employer(s)) 2020. No commercial re-use. See rights and permissions. Published by BMJ.

To cite: Azuara-Blanco A, Logan N, Strang N, et al. Br J Ophthalmol

2020;104:950-955.

\section{ABSTRACT}

Background/aims To report the protocol of a trial designed to evaluate the efficacy, safety and mechanism of action of low-dose atropine $(0.01 \%)$ eye-drops for reducing progression of myopia in UK children.

Methods Multicentre, double-masked, superiority, placebo-controlled, randomised trial. We will enrol children aged $6-12$ years with myopia of -0.50 dioptres or worse in both eyes.

We will recruit 289 participants with an allocation ratio of 2:1 (193 atropine; 96 placebo) from five centres.

Participants will instil one drop in each eye every day for 2 years and attend a research centre every 6 months. The vehicle and preservative will be the same in both study arms.

The primary outcome is SER of both eyes measured by autorefractor under cycloplegia at 2 years (adjusted for baseline). Secondary outcomes include axial length, best corrected distance visual acuity, near visual acuity, reading speed, pupil diameter, accommodation, adverse event rates and allergic reactions, quality of life (EQ$5 D-Y)$ and tolerability at 2 years. Mechanistic evaluations will include: peripheral axial length, peripheral retinal defocus, anterior chamber depth, iris colour, height and weight, activities questionnaire, ciliary body biometry and chorioretinal thickness. Endpoints from both eyes will be pooled in combined analysis using generalised estimating equations to allow for the correlation between eyes within participant. Three years after cessation of treatment, we will also evaluate refractive error and adverse events.

Conclusions The Childhood Atropine for Myopia Progression in the UK study will be the first randomised trial reporting outcomes of low-dose atropine eye-drops for children with myopia in a UK population.

Trial registration number ISRCTN99883695, NCT03690089.

\section{INTRODUCTION}

In Europe, myopia prevalence has risen dramatically over the last few decades. ${ }^{1-6}$ Myopia appears to be occurring at a younger age, and its severity has increased by an average of approximately 1 dioptre (D) among European-derived populations in one generation. ${ }^{47}$ In the UK, the proportion of myopic children has doubled in the last 50 years. ${ }^{6}$ In the USA, myopia prevalence increased from $25 \%$ to $42 \%$ in a generation. ${ }^{7}$

In the UK, most people with myopia have normal visual acuity when appropriately corrected, but myopia still has significant public health consequences from a variety of perspectives, educational, financial and psychological, as well as the risks of visual impairment. ${ }^{8-10}$ Myopia is a risk factor for myopic maculopathy, retinal detachment, cataract and glaucoma in adult life, ${ }^{911} 12$ and the risk increases with the degree of myopia. All these conditions are more challenging to treat than myopia itself, and reducing the risk for any of them requires interventions to slow myopia progression and thus decrease a child's severity of myopia in the long term rather than correct it optically with spectacles. Children with myopia also require frequent eye tests and change of spectacles that are funded primarily by taxpayers in the UK. Strategies to control progression of myopia are particularly meaningful in the context of WHO initiatives to eliminate preventable causes of blindness.

Atropine at low concentration has been shown to be safe and effective in slowing myopia progression in children of Chinese ethnicity, ${ }^{13-17}$ but its safety and effectiveness in European-derived populations has not been adequately assessed in a controlled trial. Therefore, the objective of the current study is to evaluate the efficacy, safety and mechanism of action of low-dose atropine (0.01\%) in UK children with myopia. This paper describes the protocol of the randomised controlled trial called The Childhood Atropine for Myopia Progression in the UK study (CHAMP-UK).

\section{MATERIALS AND METHODS}

This is a multicentre, randomised, double-masked, placebo-controlled, superiority trial, with 2:1 allocation of intervention and control (atropine:placebo). 


\section{Box 1 Inclusion and exclusion criteria}

\section{Inclusion criteria}

1. 6-12 years of age (at the time of consenting).

2. Myopia of -0.5 dioptre (D) or greater (spherical equivalent refractive error) in both eyes.

3. Best-corrected distance visual acuity $0.20 \log M A R$ or better in both eyes.

\section{Exclusion criteria}

1. Children with other ocular morbidities. ${ }^{*}$

2. Myopia of $-10 \mathrm{D}$ or greater in either eye.

3. Astigmatism of $2 \mathrm{D}$ or higher in either eye.

4. Amblyopia.

5. Significant health problems that can compromise the ability to attend research visits or complete the trial.

6. Other factors that may compromise the ability to attend the research appointments.

7. Parents or children with poor understanding of the English language.

8. Children enrolled in other interventional trials.

9. Allergy or hypersensitivity to atropine or excipients.

10. Prior or current use of atropine or orthokeratology contact lenses.

* Ocular comorbidities refer to any disease that can potentially compromised vision or that may require ocular surgery, including diabetes mellitus.

We followed the SPIRIT guidelines https://www.spirit-statement. org/) for reporting trials protocols.

\section{Participants}

Children will be eligible to participate in the study if they are 6-12 years of age at the time of consent, with myopia of -0.50 $\mathrm{D}$ or greater (spherical equivalent refractive error (SER)) in both eyes, best-corrected distance visual acuity (BCDVA) 0.20 logarithm of $f$ the minimum angle of resolution (logMAR) or better in both eyes, and no other significant ocular or systemic morbidities (see box 1). Children with myopia $\geq-10.00 \mathrm{D}$ or astigmatism $\geq 2.00 \mathrm{D}$ in either eye will be excluded.

\section{Setting}

Clinical research facilities from the following five academic departments of medical or optometry schools and National Health Service (NHS) Trusts in Northern Ireland, England and Scotland:

- Wellcome Trust-Wolfson Northern Ireland Clinical Research Facility: Belfast Health and Social Care Trust, Queen's University Belfast and Ulster University, Northern Ireland.

- Department of Optometry at Glasgow Caledonian University and NHS Greater Glasgow and Clyde, Scotland.

- School of Optometry at Aston University and Birmingham Women and Children's NHS Foundation Trust, England.

- Department of Vision and Hearing Sciences at Anglia Ruskin University and Cambridge University Hospitals NHS Hospital Trust, England.

- Moorfields Eye Hospital NHS Foundation Trust, England.

\section{Study interventions}

The intervention group will receive preserved $0.01 \%$ atropine sulfate eye-drops, administered at home once daily in both eyes for 2 years. The control group will receive placebo eye-drops on the same dosing schedule, with the same preservative (benzalkonium chloride $0.01 \% \mathrm{w} / \mathrm{v}$ in sterile water) and $\mathrm{pH}$. Atropine and placebo bottles will be identical, and thus participants and investigators will be masked to study group assignment.

\section{Outcomes}

The primary outcome is SER (ie, myopia severity) of both eyes after 24 months measured by autorefractor under cycloplegia, adjusted for baseline. Participants will have 1-2 drops of $1 \%$ cyclopentolate $\mathrm{HCl}$ instilled in each eye at least $20 \mathrm{~min}$ before autorefraction, and another drop will be instilled if full cycloplegia has not been achieved. The autorefractor derives an average of five readings to produce the SER. The SER (ie, the spherical power plus half the cylindrical power) will be recorded for each eye.

Secondary outcomes of both eyes include the following: axial length (measured with partial coherent interferometry), BCDVA (uniocular and binocular), uniocular and binocular near visual acuity (ETDRS), reading speed (Wilkins), pupil diameter (by autorefractometer), accommodation (using a near target), spectacle correction power, adverse event rates and allergic reactions, quality of life European Quality of Life-5 Dimensions Youth questionnaire (EQ-5D-Y) ${ }^{18}$ and tolerability at 2 years.

The mechanism through which atropine inhibits myopia remains uncertain. Possible sites of action include the lens and ciliary muscle, the sclera, choroid and the retina. ${ }^{11} \mathrm{~A}$ variety of tests will be done, according to their availability in the research units, to explore the effect of atropine on ocular anatomy and function. In experimental models, atropine reduces myopia and ocular elongation via a non-accommodative mechanism. ${ }^{19}$ However, an accommodative effect cannot be ruled out in humans. ${ }^{20}$ We will study the effect of atropine on the lens and ciliary muscle by measuring the anterior chamber depth with laser biometry and assessing changes in ciliary muscle using anterior-segment optical coherence tomography (OCT).

Altering the amount of defocus in the peripheral retina appears to be one mechanism influencing growth rate of the eye in myopia progression. ${ }^{21}$ Whether topical atropine also has some influence in the peripheral retina is unknown. Changes in peripheral eye length and off-axis measures of refraction are two ways in which the peripheral properties of the eye can be assessed.

In the posterior eye, subfoveal choroid thinning is correlated with the degree of myopia. Changes in subfoveal choroidal thickness may occur in response to imposed retinal defocus. ${ }^{22-24}$ We will study choroidal thickness using OCT. This will enable differences in choroidal thickness resulting from atropine use to be compared with normal myopic growth. We will also explore the influence of parental myopia using parents' current spectacle prescription power and time spent on different activities (table 1).

Compliance will be assessed using electronic monitoring with a MEMS device (AARDEX Group Ltd, Switzerland). The MEMS Cap is a plastic container with a screw top in which the eye-drop bottle is stored until needed for drop instillation. ${ }^{25}$ When the top is unscrewed, the device electronically records the date and time, and this is downloaded, analysed and taken as a surrogate for having administered the medication.

At 5 years after randomisation, we will post a questionnaire to participants' parents and ask for details of any possible complications and adverse events after trial completion. We will also request information from their children's optometrists regarding their eye health, visual acuity and refractive error data. 
Table 1 Diary used to record time spent indoor or outdoor

\begin{tabular}{|c|c|c|c|c|c|c|c|}
\hline Activity, to nearest whole hour & Monday & Tuesday & Wednesday & Thursday & Friday & Saturday & Sunday \\
\hline \multicolumn{8}{|l|}{ Hours indoors: classroom, music and so on } \\
\hline \multicolumn{8}{|l|}{ Hours outside: sport, training, walking/cycling to or from school } \\
\hline \multicolumn{8}{|l|}{ Hours reading, homework, video/computer/tablet/mobile phone use } \\
\hline Hours outside: sport, training, walking/cycling to or from school & & & & & & & \\
\hline
\end{tabular}

\section{Sample size}

We anticipate that the underlying progression in the control group and the effect of atropine eye-drops in a UK population will be smaller than the reported effect in Chinese populations, ${ }^{17}$ but assuming that atropine reduces the progression of myopia by at least $40 \%$, using $\mathrm{SD}=0.7$, an intraclass correlation coefficient between the two eyes of 0.9 and a variation inflation factor of 1.9, we will need 97 participants in each group. Considering a dropout rate of $15 \%$ and that $10 \%$ of recruited children will be Chinese, we will need a total of 289 participants: 193 atropine, 96 placebo (152 atropine, 76 placebo inflated by a variance inflation factor of 1.9) to detect this difference in the non-Chinese UK population with $90 \%$ power.

\section{Allocation}

Randomisation will be computer generated using a minimisation algorithm to ensure balanced allocation of participants across the two treatment groups and that each participant's allocation is fully concealed from everyone involved in recruiting them to the trial. Minimisation will be by centre, ethnic background (white/ non-white) and severity of myopia (less than $-3.00 \mathrm{D}$ in either eye/-3.00 D or greater in the eye with more severe myopia). The unit of randomisation will be the participant (not the eye). The randomisation list will be generated by sealed envelope ( sealedenvelope.com), and group allocation will only be visible to those with administrator access in the trial management team in Northern Ireland Clinical Trials Unit (NICTU). Local researchers will access the automated randomisation system to obtain the kit number for each participant.

\section{Adverse events/safety reporting}

Serious adverse events related to participants' participation in the trial are reported in accordance with the guidance from The European Clinical Trials Directive 2001/20/EC (https://ec. europa.eu/health/human-use/clinical-trials/directive en).

\section{Timeline of procedures}

Table 2 displays the timing of the trial's outcome measurements. Participants will attend a research centre every 6 months $( \pm 2$ weeks) across the 2-year follow-up as illustrated in figure 1 (see table 2 for details). Following completion of the 2 -year trial, participants will then again be contacted at the 5 -year time point (3 years after cessation of eye-drops) to evaluate their refractive error and possible adverse events.

\section{Recruitment strategy}

We will ask community optometrists and paediatric ophthalmologists to inform parents of children with myopia about the trial. We will aim to recruit approximately 60 participants per centre.

\section{Data collection}

The chief investigator (CI) and NICTU will provide training to site staff on trial processes and procedures, including the completion of the clinical research form (CRF) and data collection through investigator meetings and site initiation visits. All data for an individual participant will be collected by the local principal investigator (PI) or designee and recorded in the CRF for the study. Participant identification on the CRF will be through

\begin{tabular}{|c|c|c|c|c|c|}
\hline & Baseline & 6 months & 12 months & 18 months & 24 months \\
\hline Adverse events & & $\checkmark$ & $\checkmark$ & $\boldsymbol{\sim}$ & $\checkmark$ \\
\hline Tolerability & & $\boldsymbol{\nu}$ & $\checkmark$ & $\checkmark$ & $\checkmark$ \\
\hline EQ-5D-Y Questionnaire & $\checkmark$ & $\checkmark$ & $\checkmark$ & $\boldsymbol{V}$ & $\checkmark$ \\
\hline Activities questionnaire - to be sent home with participant for completion & $\boldsymbol{V}$ & $\checkmark$ & $\boldsymbol{V}$ & $\boldsymbol{V}$ & $\checkmark$ \\
\hline Best-corrected VA (logMAR ETDRS) & $\checkmark$ & $\checkmark$ & $\checkmark$ & $\checkmark$ & $\checkmark$ \\
\hline Near VA (near logMAR ETDRS) & $\checkmark$ & $\checkmark$ & $\checkmark$ & $\boldsymbol{V}$ & $\checkmark$ \\
\hline Iris colour & $\checkmark$ & & & & \\
\hline Reading speed (Wilkins Rate of Reading Test) & $\checkmark$ & $\checkmark$ & $\checkmark$ & $\checkmark$ & $\checkmark$ \\
\hline Pupil diameter prior to cycloplegia (autorefractor) & $\checkmark$ & $\checkmark$ & $\checkmark$ & $\checkmark$ & $\checkmark$ \\
\hline Accommodation (autorefractor) & $\checkmark$ & $\checkmark$ & $\checkmark$ & $\checkmark$ & $\checkmark$ \\
\hline Peripheral retinal defocus (autorefractor) & $\checkmark$ & & $\checkmark$ & & $\checkmark$ \\
\hline Anterior chamber depth (laser biometer) & $\checkmark$ & $\checkmark$ & $\checkmark$ & $\checkmark$ & $\checkmark$ \\
\hline Cycloplegic refractive error (autorefractor) & $\checkmark$ & $\checkmark$ & $\boldsymbol{V}$ & $\checkmark$ & $\checkmark$ \\
\hline Ciliary body biometry (AS-OCT)* & $\checkmark$ & & $\checkmark$ & & $\checkmark$ \\
\hline Central axial length (laser biometer) & $\checkmark$ & $\checkmark$ & $\checkmark$ & $\checkmark$ & $\checkmark$ \\
\hline Peripheral axial length (laser biometer) & $\checkmark$ & & $\checkmark$ & & $\checkmark$ \\
\hline Chorioretinal thickness (SD-OCT) & $\checkmark$ & $\checkmark$ & $\checkmark$ & $\checkmark$ & $\checkmark$ \\
\hline
\end{tabular}

$\checkmark \boldsymbol{V}$ means before and after cycloplegia.

*If instrumentation is available.

$\mathrm{VA}$, visual acuity. 


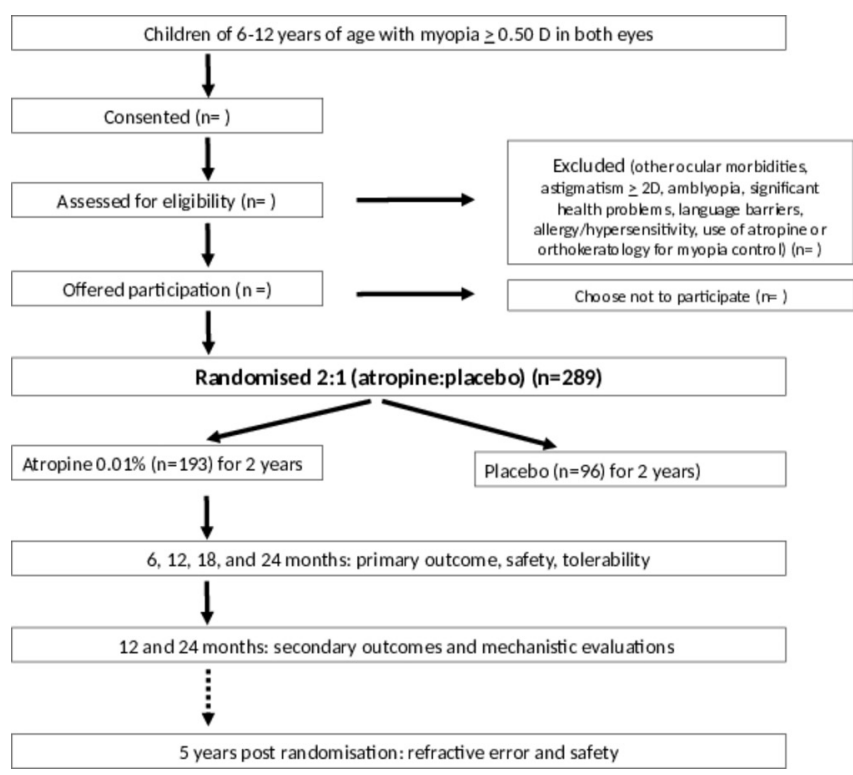

Figure 1 Study flow chart with outcome timeline.

their unique trial identifier, allocated at the time of recruitment. Data will be collected and recorded on the CRF and questionnaires by the local PI or designee. Case report forms and questionnaires will be submitted to the NICTU in a timely manner.

\section{Data management}

Study data will be entered onto a web-based Clinical Trial Database and processed electronically as per NICTU standard operating procedures and the study specific data management plan. Data queries will be generated for site staff as required to clarify data or request missing information. All queries will be responded to and resolved within the study database. Any amended information will then be entered in the study database. All study documentation (including participant medical records) and data will be archived as per regulatory requirements and those responsible for archiving will be noted on the sponsor delegation framework.

\section{Statistical analysis}

The primary analysis will be performed based on the intention to treat principle. A p value $<0.05$ is considered as statistically significant. Baseline characteristics will be summarised as mean and SD, median and IQR or numbers and proportions (\%) as appropriate, depending on the scale of measurement and distribution.

Endpoints from both eyes will be pooled in combined analysis using generalised estimating equations to allow for the correlation between eyes within participant. Difference in the myopia progression and other continuous outcomes between the atropine and control groups will also be tested for significance using independent t-test. Analysis of covariance will be performed to adjust for baseline characteristics and other covariates. Fisher's exact test will be used to test the difference in the proportions between the groups for the categorical variables. Exploratory subgroup analyses will be performed on the primary outcome using 99\% CIs and interaction terms (treatment group by subgroup) for the following subgroups: age (6-9 and 10-12 years at randomisation), ethnic background (white vs non-white) and severity of myopia (less than $-3 \mathrm{D}$ in either eye vs $-3 \mathrm{D}$ or greater myopia). Sensitivity analyses will assess the impact of missing data for the primary outcome by imputing extreme values (lowest and highest). A detailed Statistical Analysis Plan will be completed before the final analysis is started.

\section{Monitoring}

On-site monitoring will be an ongoing activity from the time of initiation until trial closeout and will comply with the principles of Good Clinical Practice (GCP). On-site monitoring visits during the trial will check the accuracy of entries on CRFs against the source documents, the adherence to the protocol, study procedures and GCP. The local PI or designee will ensure that access to all trial related documents including source documents (to confirm their consistency with CRF entries) are available during monitoring visits. The extent of source data verification will be documented in the monitoring plan.

\section{Ethics and governance}

The trial will comply with the principles of GCP, the requirements and standards set out by the EU Directive 2001/20/EC and the applicable regulatory requirements in the UK, the Medicines for Human Use (Clinical Trials) Regulations 2004 and subsequent amendments and the UK Policy Framework for Health and Social Care Research. Local NHS Research and Development approvals will be obtained prior to commencing the trial at the participating sites. An independent data and safety monitoring committee will oversee the trial. A Clinical Trial Authorisation was obtained from the Medicines for Human Use Regulatory Authority before the start of the trial.

\section{Protocol compliance}

A protocol deviation is defined as an incident that deviates from the normal expectation of a particular part of the trial process. Any deviations from the protocol will be fully documented on the protocol deviation form in the CRF.

A serious breach is defined as a deviation from the trial protocol or GCP, which is likely to effect to a significant degree:

a. The safety or physical or mental integrity of the subjects of the trial or

b. The scientific value of the trial.

Protocol compliance will be monitored by the NICTU.

\section{Protocol amendments}

Changes to the protocol will require regulatory authority/ethics committee approval/favourable opinion prior to implementation.

\section{Participant confidentiality}

All study reports and communication regarding the study will identify the participants by the assigned unique trial identifier only. Computers where information will be stored will be password protected. Participant confidentiality will be maintained at every stage, and identifying data will not be made publicly available to the extent permitted by the applicable laws and regulations.

\section{Post-trial care}

Administration of study eye-drops will stop after 24 months of trial participation. At the end of the trial, participants' myopia will be managed in accordance with standard clinical practice.

\section{Dissemination policy}

The final study data report will be provided by the trial statistician. It is anticipated that the study findings will be published in peer-reviewed journals, and these articles will be led by the CI. 
The CI will secure a searchable compendium of these publications and make the results readily accessible to the public and healthcare professionals. In addition, study findings may be presented at both national and international meetings and to appropriate patient groups.

\section{Data sharing statement}

Requests for data sharing will be reviewed on a case-by-case basis by the CI and Trial Management Group. We will share trial data with the CI of the Myopia Outcome Study of Atropine in Children (MOSAIC) trial (ISRCTN36732601) and the Western Australian Atropine for the treatment of myopia (WA-ATOM) trial (ACTRN12617000598381) to facilitate prospective individual participant data meta-analysis once the results of the CHAMP-UK trial are accepted for publication. MOSAIC and WA-ATOM are placebo-controlled trials evaluating $0.01 \%$ atropine eye-drops. WA-ATOM will enrol 150 children aged 6-16 years with progressive myopia. MOSAIC will enrol 250 children aged 6-16 years with progressive myopia (phase 1). All participants initially assigned to the placebo $(n=83)$ crossover to the intervention arm of the study for phase 2, and from month 24 to 36 , instil $0.01 \%$ atropine eye-drops in both eyes once nightly.

\section{DISCUSSION}

Myopia typically starts to develop in childhood, and although the vision can be corrected with glasses, contact lenses or surgery, myopic eyes have an increased risk of developing comorbidities such as glaucoma, cataract, retinal detachment and choroidal neovascularisation at the macula. ${ }^{11}$ Importantly, the risks of associated comorbidity and visual loss are associated with the degree of myopia and cannot be reduced with optical correction alone. Myopia is more prevalent in East Asia. Recent epidemiological studies show increasing rates among adolescents in European populations and suggest myopia is occurring at an earlier age than in previous generations. ${ }^{3-5}$

Myopia usually progresses faster at younger ages, but myopia onset, progression and stabilisation vary widely among individuals and are influenced by a range of variables including environment, lifestyle, parental refractive history and ethnicity. ${ }^{426}$

A number of interventions to reduce the progression of myopia have been investigated. Multifocal lenses and under-correction of myopic refractive error have at best a weak effect on myopia correction. While orthokeratology and peripheral defocus contact lenses may have some effect on axial length progression, atropine, even at low doses, appears to be the most effective intervention in Asian children to reduce progression of myopic refractive error. ${ }^{27} 28$ Low-dose atropine is now widely used in some East Asian countries for treating children with myopia but has not been tested in European populations.

Atropine is an anticholinergic agent that is relatively selective for muscarinic receptors. Topical use of high-concentration atropine $(0.5 \%-1.0 \%)$ causes pupil dilatation by blocking the muscarinic receptors in the pupillary sphincter musculature and reduces or paralyses contraction of the ciliary muscle. Both of these result in adverse effects, for example, photophobia and blurred near (reading) vision that are highly undesirable in a school-age population. Additionally, cessation of topical highconcentration atropine for myopia control has been associated with rapid myopia progression towards original, untreated levels ('myopia rebound'). ${ }^{15}$

The effectiveness of low-dose atropine (three different concentrations) in children of Chinese race has been evaluated. ${ }^{13-16}$ The lowest tested dose of $0.01 \%$ was associated with better tolerability and efficacy, with minimum rebound effect. ${ }^{14}$ A systematic review and network meta-analysis has confirmed muscarinic antagonists as the most effective interventions for myopia control in children of Chinese ethnicity. ${ }^{27}$ However, there is limited evidence from European populations on atropine effectiveness in controlling myopia progression. We will also evaluate the possible mechanisms of action of atropine and gather information regarding central and peripheral axial length, accommodation, lens position, ciliary body biometry, chorioretinal thickness and daily activities.

\section{Author affiliations}

${ }^{1}$ Centre for Public Health, Queen's University Belfast School of Medicine Dentistry and Biomedical Sciences, Belfast, UK

${ }^{2}$ School of Life \& Health Sciences, Aston University, Birmingham, UK

${ }^{3}$ Department of Vision Sciences, Glasgow Caledonian University, Glasgow, UK

${ }^{4}$ School of Biomedical Sciences, Ulster University, Coleraine, UK

${ }^{5}$ Department of Optometry and Ophthalmic Dispensing, Anglia Ruskin University,

Cambridge, UK

${ }^{6}$ NICTU, Belfast Health and Social Care Trust, Belfast, UK

${ }^{7}$ Department of Pharmacy, Belfast Health and Social Care Trust, Belfast, UK

${ }^{8}$ Institute of Translational Medicine, University of Liverpool, Liverpool, UK

${ }^{9}$ Optometry Department, Dublin Institute of Technology, Dublin, Ireland

${ }^{10}$ Department of Ophthalmology, Childrens University Hospital, Dublin, Ireland

${ }^{11}$ Centre for Ophthalmology and Visual Science, University of Western Australia, Lions Eye Institute, Perth, Western Australia, Australia

${ }^{12}$ Department of Twin Research and Genetic Epidemiology, King's College London, London, UK

${ }^{13}$ Department of Preventive Ophthalmology, Zhongshan Ophthalmic Center,

Guangdong, China

${ }^{14}$ Orbis International, New York, United States

\section{Twitter Nicola Logan @drNicolaLogan and Ruth Hogg @ruth_hogg}

Acknowledgements Trial steering committee: Richard Wormald, John Buchan, Hetal Buckhurst, Hema Radhakrishnan, Helen Normington. Data and safety monitoring committee: Jennifer Burr, David Cooper and Jeremy Guggenheim.

Collaborators Annegret Dahlmann-Noor.

Funding The trial is funded by a grant awarded by National Institute for Health Research Efficacy and Mechanism Evaluation (project 15/48/59). CHAMP-UK is registered (ISRCTN99883695, NCT03690089). The Belfast Health and Social Care Trust sponsors the trial and provides the necessary trial insurance.

Competing interests NL: research funding from CooperVision, Essilor and Zeiss. Patient consent for publication Not required.

Ethics approval CHAMP-UK has been reviewed and approved by a Research Ethics Committee (18/NI/0164).

Provenance and peer review Not commissioned; externally peer reviewed.

Data availability statement Data are available on request.

Author note Acronym: The Childhood Atropine for Myopia Progression in the UK study (CHAMP-UK).

\section{ORCID iDs}

Augusto Azuara-Blanco http://orcid.org/0000-0002-4805-9322

Nicola Logan http://orcid.org/0000-0002-0538-9516

Ruth Hogg http://orcid.org/0000-0001-9413-2669

James J Loughman http://orcid.org/0000-0003-3130-8991

Samantha Sze-Yee Lee http://orcid.org/0000-0001-6635-1098

Christopher Hammond http://orcid.org/0000-0002-3227-2620

Nathan Congdon http://orcid.org/0000-0001-9866-3416

\section{REFERENCES}

1 Rudnicka AR, Kapetanakis VV, Wathern AK, et al. Global variations and time trends in the prevalence of childhood myopia, a systematic review and quantitative meta-analysis: implications for aetiology and early prevention. Br J Ophthalmol 2016;100:882-90.

2 Breslin KMM, O'Donoghue L, Saunders KJ. A prospective study of spherical refractive error and ocular components among Northern Irish schoolchildren (the NICER study). Invest Ophthalmol Vis Sci 2013;54:4843-50.

3 Williams KM, Hysi PG, Nag A, et al. Age of myopia onset in a British population-based twin cohort. Ophthalmic Physiol Opt 2013;33:339-45. 
4 Williams KM, Bertelsen G, Cumberland P, et al. Increasing prevalence of myopia in Europe and the impact of education. Ophthalmology 2015;122:1489-97.

5 Williams KM, Verhoeven VJM, Cumberland P, et al. Prevalence of refractive error in Europe: the European eye epidemiology (E3) Consortium. Eur J Epidemiol 2015;30:305-15.

6 McCullough SJ, O'Donoghue L, Saunders KJ. Six year refractive change among white children and young adults: evidence for significant increase in myopia among white UK children. PLoS One 2016;11:e0146332.

7 Vitale S, Ellwein L, Cotch MF, et al. Prevalence of refractive error in the United States, 1999-2004. Arch Ophthalmol 2008;126:1111-9.

$8 \mathrm{Ma} \mathrm{X}$, Zhou Z, Yi H, et al. Effect of providing free glasses on children's educational outcomes in China: cluster randomized controlled trial. BMJ 2014;349:g5740.

9 Zheng Y-F, Pan C-W, Chay J, et al. The economic cost of myopia in adults aged over 40 years in Singapore. Invest Ophthalmol Vis Sci 2013;54:7532-7.

10 Tideman JWL, Snabel MCC, Tedja MS, et al. Association of axial length with risk of uncorrectable visual impairment for Europeans with myopia. JAMA Ophthalmol 2016;134:1355-63.

11 Flitcroft DI. The complex interactions of retinal, optical and environmental factors in myopia aetiology. Prog Retin Eye Res 2012;31:622-60.

12 Marcus MW, de Vries MM, Junoy Montolio FG, et al. Myopia as a risk factor for open-angle glaucoma: a systematic review and meta-analysis. Ophthalmology 2011;118:1989-94.

13 Chia A, Chua W-H, Cheung Y-B, et al. Atropine for the treatment of childhood myopia: safety and efficacy of $0.5 \%, 0.1 \%$, and $0.01 \%$ doses (Atropine for the Treatment of Myopia 2). Ophthalmology 2012;119:347-54.

14 Chia A, Chua W-H, Wen L, et al. Atropine for the treatment of childhood myopia: changes after stopping atropine $0.01 \%, 0.1 \%$ and $0.5 \%$. Am J Ophthalmol 2014:157:451-7.

15 Chia A, Lu Q-S, Tan D. Five-Year Clinical Trial on Atropine for the Treatment of Myopia 2: Myopia Control with Atropine 0.01\% Eyedrops. Ophthalmology 2016:123:391-9.

16 Yam JC, Jiang Y, Tang SM, et al. Low-Concentration Atropine for Myopia Progression (LAMP) study: a randomized, double-blinded, placebo-controlled Trial of 0.05\%,
$0.025 \%$, and $0.01 \%$ atropine eye drops in myopia control. Ophthalmology 2019;126:113-24

17 Li S-M, Wu S-S, Kang M-T, et al. Atropine slows myopia progression more in Asian than white children by meta-analysis. Optom Vis Sci 2014;91:342-50.

18 Wille N, Badia X, Bonsel G, et al. Development of the EQ-5D-Y: a child-friendly version of the EQ-5D. Qual Life Res 2010;19:875-86. 18.

19 McBrien NA, Moghaddam HO, Reeder AP. Atropine reduces experimental myopia and eye enlargement via a nonaccommodative mechanism. Invest Ophthalmol Vis Sci 1993;34:205-15.

20 Schmid KL, Strang NC. Differences in the accommodation stimulus response curves of adult myopes and emmetropes: a summary and update. Ophthalmic Physiol Opt 2015;35:613-21.

21 Smith EL, Hung L-F, Huang J. Relative peripheral hyperopic defocus alters central refractive development in infant monkeys. Vision Res 2009;49:2386-92.

22 Chiang ST-H, Phillips JR, Backhouse S. Effect of retinal image defocus on the thickness of the human choroid. Ophthalmic Physiol Opt 2015;35:405-13.

23 Read SA, Collins MJ, Vincent SJ, et al. Choroidal thickness in myopic and nonmyopic children assessed with enhanced depth imaging optical coherence tomography. Invest Ophthalmol Vis Sci 2013;54:7578-86.

24 Read SA, Alonso-Caneiro D, Vincent SJ, et al. Longitudinal changes in choroidal thickness and eye growth in childhood. Invest Ophthalmol Vis Sci 2015;56:3103-12.

25 Boland MV, Chang DS, Frazier T, et al. Electronic monitoring to assess adherence with once-daily glaucoma medications and risk factors for nonadherence: the automated dosing reminder study. JAMA Ophthalmol 2014;132:838-44.

26 Donovan L, Sankaridurg P, Ho A, et al. Myopia progression in Chinese children is slower in summer than in winter. Optom Vis Sci 2012;89:1196-202.

27 Walline JJ, Lindsley K, Vedula SS, et al. Interventions to slow progression of myopia in children. Cochrane Database Syst Rev 2011:CD004916.

28 Huang J, Wen D, Wang Q, et al. Efficacy comparison of 16 interventions for myopia control in children: a network meta-analysis. Ophthalmology 2016;123:697-708. 